Proyecciones Journal of Mathematics

Vol. 37, No 1, pp. 103-118, March 2018.

Universidad Católica del Norte

Antofagasta - Chile

\title{
Cosine families of operators in a class of Fréchet spaces
}

\author{
R. Ameziane Hassani \\ Université S. M. B. A., Maroc \\ A. Blali \\ Université S. M. B. A., Maroc \\ A. EL Amrani \\ Université S. M. B. A., Maroc \\ and \\ K. Moussaouja \\ Université S. M. B. A., Maroc \\ Received: March 2017. Accepted: March 2017
}

\begin{abstract}
M. sova [10] proved that the infinitesimal generator of all uniformly continuous cosine family, of operators in Banach space, is a bounded operator. We show by counter-example that the result mentioned above is not true in general on Fréchet spaces, and we prove that the infinitesimal generator of an uniformly continuous cosine family of operators in a class of Fréchet spaces (quojection) is necessarily continuous.
\end{abstract}

Keywords : Strongly continuous cosine families. Semi-groups of operators. Locally convex space. Quojection Fréchet space. 


\section{Introduction}

A strongly continuous cosine family, of bounded operators in Banach space $X$, appear as solution of the abstract Cauchy problem of second order [2][5] :

$$
U^{\prime \prime}=A U, \quad U(0)=x, \quad \text { and } \quad U^{\prime}(0)=0 .
$$

A link between the family $\{C(t)\}_{t \in \mathbf{R}}$ and the operator $A$ is given by the Laplace transform : $\lambda\left(\lambda^{2} I-A\right)^{-1}=\int_{0}^{\infty} e^{-\lambda s} C(s) d s$, and $A$ is called the infinitesimal generator of the cosine family $\{C(t)\}_{t \in \mathbf{R}}[2][4]$.

We know, in classical theory, that the infinitesimal generator $A$ of an uniformly continuous cosine family $\{C(t)\}_{t \in \mathbf{R}}$, of bounded operators in $X$ (Banach space), is a bounded operator [4][10], moreover for all $t \in \mathbf{R}$ we have :

$$
C(t)=\operatorname{Cosh}(t \sqrt{A})=\sum_{n=0}^{\infty} \frac{t^{2 n}}{(2 n) !} A^{n} .
$$

In section 1, We give the definition of cosine family of operators in locally convex spaces, and some propositions important for our results.

An example, of uniformly continuous cosine family, of operators in a Fréchet space, whose infinitesimal generator is a closed operator, densely defined and not continuous on the space everywhere, is given in the second section; and we demonstrate that in the case where the Fréchet space is quojection [Definition 3.1], the infinitesimal generator of all uniformly continuous cosine family of operators is necessarily continuous.

\section{Cosine families of operators in locally convex space :}

Let $X$ be a locally convex Hausdorff space, and $\Gamma_{X}$ a system of continuous semi-norms determining the topology of $X$. The strong topology $\tau_{s}$ in the space $\mathcal{L}(X)$, of all continuous linear operators from $X$ into itself, is determined by the family of seminorms :

$$
q_{x}(S)=q(S x), \quad S \in \mathcal{L}(X),
$$

for each $x \in X$ and $q \in \Gamma_{X}, \mathcal{L}(X)$ equipped with this topology is noted $\mathcal{L}_{s}(X)$. 
Let $B(X)$ the collection of all bounded subsets of $X$. The topology $\tau_{b}$ of uniform convergence on the elements of $B(X)$ is defined by the family of semi-norms :

$$
q_{B}(S)=\sup _{x \in B} q(S x), \quad S \in \mathcal{L}(X),
$$

for each $B \in B(X)$ and $q \in \Gamma_{X}, \mathcal{L}(X)$ equipped with this topology is noted $\mathcal{L}_{b}(X)$.

Definition 1. Let $\{C(t)\}_{t \in \mathbf{R}} \subseteq \mathcal{L}(X)$ be a family of operators verifying the following properties:

1. $C(0)=I$.

2. $2 C(t) C(s)=C(t+s)+C(t-s), \forall s, t \in \mathbf{R}$.

- We say that $\{C(t)\}_{t \in \mathbf{R}}$ is a strongly continuous cosine family if :

$$
C(t) \longrightarrow C\left(t_{0}\right) \text { in } \mathcal{L}_{s}(X), \text { as } t \longrightarrow t_{0}, \quad \forall t_{0} \in \mathbf{R} .
$$

- we say that $\{C(t)\}_{t \in \mathbf{R}}$ is an uniformly continuous cosine family if :

$$
C(t) \longrightarrow C\left(t_{0}\right) \text { in } \mathcal{L}_{b}(X), \text { as } t \longrightarrow t_{0}, \quad \forall t_{0} \in \mathbf{R} .
$$

Definition 2. Let $X$ be a sequentially complete locally convex Hausdorff space and $\{C(t)\}_{t \in \mathbf{R}}$ be a strongly continuous cosine family on $X$.

Let $A$ the operator defined on $D(A)$ by :

$A x=\lim _{t \longrightarrow 0} \frac{2}{t^{2}}(C(t) x-x)$, where $D(A)=\left\{x \in X / \lim _{t \longrightarrow 0} \frac{2}{t^{2}}(C(t) x-x)\right.$ exists in $\left.X\right\}$.

$A$ is called the infinitesimal generator of $\{C(t)\}_{t \in \mathbf{R}}$.

Remark 1. - According to 2. of the Definition 1., we have $C($.$) is even.$

Indeed, for $t=0$ we have $2 C(s)=C(s)+C(-s)$ which implies that $C(s)=C(-s), \forall s \in \mathbf{R}$.

- For all $t, s \in \mathbf{R}$, we have $C(t) C(s)=C(s) C(t)$.

Indeed, $2 C(t) C(s)=C(t+s)+C(t-s)$

$=C(s+t)+C(s-t)=2 C(s) C(t), \quad \forall t, s \in \mathbf{R}$. 
We always denote by $X$ a sequentially complete locally convex Hausdorff space, and $\Gamma_{X}$ a system of continuous semi-norms determining the topology of $X$. We recall that a family $H \subset \mathcal{L}(X)$ is called equicontinuous if for all neighborhood $\mathbf{U}$ of 0 , there exists a neighborhood $\mathbf{V}$ of 0 such that $T(\mathbf{V}) \subseteq \mathbf{U}, \forall T \in H[6]$; and we say that a family $\{C(t)\}_{t \in \mathbf{R}} \subset \mathcal{L}(X)$ is locally equicontinuous, if for all $s \in \mathbf{R}^{+}$, the set $\{C(t), \quad-s \leq t \leq s\}$ is equicontinuous.

Proposition 1. Let $\{C(t)\}_{t \in \mathbf{R}}$ be a strongly continuous cosine family on $X$. Then for all $x \in X$ and $t \in \mathbf{R}$ we have :

$$
\lim _{h \longrightarrow 0} \frac{2}{h^{2}} \int_{t}^{t+h}(t+h-s) C(s) x d s=C(t) x .
$$

Proof. Let $t \in \mathbf{R}, x \in X$ and $p \in \Gamma_{X}$. Thus for all $h \in \mathbf{R}_{+}^{*}$ we have :

$$
\begin{aligned}
& p\left(\frac{2}{h^{2}} \int_{t}^{t+h}(t+h-s) C(s) x d s-C(t) x\right) \\
& =p\left(\frac{2}{h^{2}} \int_{t}^{t+h}(t+h-s)(C(s) x-C(t) x) d s\right) \\
& \leq \frac{2}{h^{2}} \int_{t}^{t+h}(t+h-s) p(C(s) x-C(t) x) d s \\
& \leq \frac{2}{h^{2}} \int_{t}^{t+h}(t+h-s) d s \sup _{s \in[t, t+h]} p(C(s) x-C(t) x) \\
& \leq \sup _{s \in[t, t+h]} p(C(s) x-C(t) x) .
\end{aligned}
$$

Since $C(t) x$ is continuous on $\mathbf{R}$, Then $\sup _{s \in[t, t+h]} p(C(s) x-C(t) x) \longrightarrow$ 0 , as $h \longrightarrow 0$.

Similarly, for $h \in \mathbf{R}_{-}^{*}$ we have $\sup _{s \in[t+h, t]} p(C(s) x-C(t) x) \longrightarrow 0$, as $h \longrightarrow 0$.

Hence the result.

Remark 2. If $\{C(t)\}_{t \in \mathbf{R}}$ is an uniformly continuous cosine family, then for all $t \in \mathbf{R}$ we have:

$$
\frac{2}{h^{2}} \int_{t}^{t+h}(t+h-s) C(s) d s \longrightarrow C(t) \text { in } \mathcal{L}_{b}(X), \text { as } h \longrightarrow 0 .
$$

Corollary 1. Let $\{C(t)\}_{t \in \mathbf{R}}$ be a strongly continuous cosine family on $X$, and $A$ its infinitesimal generator. For all $t \in \mathbf{R}$, and $x \in X$ we have :

$$
\int_{0}^{t}(t-s) C(s) x d s \in D(A), \text { and } A \int_{0}^{t}(t-s) C(s) x d s=C(t) x-x .
$$


Proof. Let $h \in \mathbf{R}^{*}$, for all $x \in X$ we have :

$$
\begin{aligned}
& \frac{2}{h^{2}}(C(h)-I) \int_{0}^{t}(t-s) C(s) x d s \\
& =\frac{2}{h^{2}} \int_{0}^{t}(t-s) C(h) C(s) x d s-\frac{2}{h^{2}} \int_{0}^{t}(t-s) C(s) x d s \\
& =\frac{1}{h^{2}} \int_{0}^{t}(t-s) C(s+h) x d s+\frac{1}{h^{2}} \int_{0}^{t}(t-s) C(s-h) x d s \\
& -\frac{2}{h^{2}} \int_{0}^{t}(t-s) C(s) x d s \\
& =\frac{1}{h^{2}} \int_{h}^{t+h}(t+h-s) C(s) x d s+\frac{1}{h^{2}} \int_{-h}^{t-h}(t-h-s) C(s) x d s \\
& -\frac{2}{h^{2}} \int_{0}^{t}(t-s) C(s) x d s \\
& =\frac{1}{h^{2}} \int_{t}^{t+h}(t+h-s) C(s) x d s+\frac{1}{h^{2}} \int_{t}^{t-h}(t-h-s) C(s) x d s \\
& -\frac{1}{h^{2}} \int_{0}^{h}(h-s) C(s) x d s-\frac{1}{h^{2}} \int_{0}^{-h}(-h-s) C(s) x d s .
\end{aligned}
$$

According to proposition 1. we obtain $\int_{0}^{t}(t-s) C(s) x d s \in D(A)$, and

$$
A \int_{0}^{t}(t-s) C(s) x d s=C(t) x-x .
$$

Proposition 2. Let $\{C(t)\}_{t \in \mathbf{R}}$ be a strongly continuous cosine family on $X$, and $A$ its infinitesimal generator.

Then $D(A)$ is dense in $X$.

Proof. Let $\left(h_{n}\right)_{n \in \mathbf{N}} \subseteq \mathbf{R}^{*}$ be a sequence such that $h_{n} \longrightarrow 0$, as $n \longrightarrow \infty$.

Let $x \in X$, put :

$$
x_{n}=\frac{2}{h_{n}^{2}} \int_{0}^{h_{n}}\left(h_{n}-s\right) C(s) x d s
$$

According to corollary 1 . we have $\left(x_{n}\right)_{n} \subseteq D(A)$, and for $t=0$ in the Proposition 1 . we have $x_{n} \longrightarrow x$, as $n \longrightarrow \infty$.

Then $D(A)$ is dense in $X$.

Proposition 3. If $X$ is tonnelé, Then every strongly continuous cosine family $\{C(t)\}_{t \in \mathbf{R}}$ on $X$ is locally equicontinuous. 
Proof. Let $s>0$, and $\mathcal{U}$ be any convex circular closed neighborhood of 0 , then $\mathcal{V}=\bigcap_{-s \leq t \leq s}(C(t))^{-1}(\mathcal{U})$ is a convex circular closed set.

Let $x \in X$, the set $\{C(t) x,-s \leq t \leq s\}$ is bounded in $X$, because the strongly continuity of the family $\{C(t)\}_{t \in \mathbf{R}}$ on $X$; since $\mathcal{U}$ is a neighborhood of $0, \exists \lambda \geq 0$ such that : $\{C(t) x,-s \leq t \leq s\} \subseteq \lambda \mathcal{U}$. Which implies that $x \in \lambda \mathcal{V}$, this means that $\mathcal{V}$ is absorbing, and $C(t)(\mathcal{V}) \subseteq \mathcal{U}, \forall t \in[-s, s]$.

Since $X$ is tonnelé, $\mathcal{V}$ is a neighborhood of 0 , which means that $\{C(t) x,-s \leq$ $t \leq s\}$ is equicontinuous.

Proposition 4. Let $\{C(t)\}_{t \in \mathbf{R}}$ be a locally equicontinuous strongly continuous cosine family on $X$. Let $x$ and $y$ in $X$, then :

$x \in D(A)$, and $A x=y$, if and only if, $C(t) x-x=\int_{0}^{t}(t-s) C(s) y d s . \quad \forall t \in$ R.

Proof. $\Leftarrow$ Evident (proposition $\mathbf{1})$.

$\Rightarrow$ Let $x, y \in X$, such that $A x=y$.

Firstly, we have for all $t \in \mathbf{R}, \int_{0}^{t}(t-s) C(s) x d s \in D(A)$ and :

$$
C(t) x-x=A \int_{0}^{t}(t-s) C(s) x d s=\lim _{h \rightarrow 0} \int_{0}^{t}(t-s) C(s) \frac{2}{h^{2}}(C(h) x-x) d s .
$$

Let $t \in \mathbf{R}_{+}^{*}$, since $\{C(t)\}_{t \in \mathbf{R}}$ is locally equicontinuous on $X, \forall p \in \Gamma_{X}$, $\exists q \in \Gamma_{X}, \exists M \geq 0$ such that :

$$
p(C(t) x) \leq M q(x), \quad \forall s \in[-2 t, 2 t] .(*)
$$

Then, for all $h \in \mathbf{R}^{*}$, with $|h| \leq t$

$$
\begin{aligned}
& p\left(\int_{0}^{t}(t-s) C(s) \frac{2}{h^{2}}(C(h) x-x) d s\right) \\
& =p\left(\frac{1}{h^{2}} \int_{t}^{t-h}(t-h-s) C(s) x d s+\frac{1}{h^{2}} \int_{t}^{t+h}(t+h-s) C(s) x d s\right. \\
& \left.-\frac{2}{h^{2}} \int_{0}^{h}(h-s) C(s) x d s\right) \\
& \leq 3 \sup _{[-2 t, 2 t]} p(C(s) x) .
\end{aligned}
$$

According to (*), $\forall p \in \Gamma_{X}, \exists q \in \Gamma_{X}, \exists M \geq 0$ we have :

$$
p\left(\int_{0}^{t}(t-s) C(s) \frac{2}{h^{2}}(C(h) x-x) d s\right) \leq 3 M q(x) .
$$


Therefore,

$$
\begin{gathered}
\lim _{h \longrightarrow 0^{+}} \int_{0}^{t}(t-s) C(s) \frac{2}{h^{2}}(C(h) x-x) d s \\
=\int_{0}^{t}(t-s) C(s) \lim _{h \longrightarrow 0} \frac{2}{h^{2}}(C(h) x-x) d s=\int_{0}^{t}(t-s) C(s) y d s .
\end{gathered}
$$

Hence,

$$
C(t) x-x=\int_{0}^{t}(t-s) C(s) y d s, \quad t \in \mathbf{R} .
$$

Corollary 2. The infinitesimal generator of all locally equicontinuous strongly continuous cosine family on $X$ is closed.

Proof. Let $\left(x_{n}\right)_{n \in \mathbf{N}} \subseteq D(A)$ such that $\lim _{n \longrightarrow \infty} x_{n}=x$, and $\lim _{n \longrightarrow \infty} A x_{n}=$ $y$.

Let $t \in \mathbf{R}$ fix, then we have $C(t) x_{n}-x_{n}=\int_{0}^{t}(t-s) C(s) A x_{n} d s, \forall n \in \mathbf{N}$.

Since $C(t) \in \mathcal{L}(X), \lim _{n \longrightarrow \infty}\left(C(t) x_{n}-x_{n}\right)=C(t) x-x$.

As $\{C(t)\}_{t \in \mathbf{R}}$ is locally equicontinuous, $\lim _{n \rightarrow \infty} \int_{0}^{t}(t-s) C(s) A x_{n} d s=$ $\int_{0}^{t}(t-s) C(s) y d s$.

Indeed, let $p \in \Gamma_{X}$ and $t \in \mathbf{R}^{+}$, we have :

$$
\begin{aligned}
p\left(\int_{0}^{t}(t-s) C(s) A x_{n} d s-\int_{0}^{t}(t-s) C(s) y d s\right) & =p\left(\int_{0}^{t}(t-s) C(s)\left(A x_{n}-y\right) d s\right) \\
& \leq \frac{t^{2}}{2} \sup _{0 \leq s \leq t} p\left(C(t)\left(A x_{n}-y\right)\right) .
\end{aligned}
$$

Since $\{C(s)\}_{s \in \mathbf{R}}$ is locally equicontinuous, $\exists q \in \Gamma_{X}, \exists M>0$ such that :

$$
p(C(t) x) \leq M q(x), \quad \forall x \in X \text { et } \forall s \in[-t, t] .
$$

Consequently,

$$
p\left(\int_{0}^{t}(t-s) C(s) A x_{n} d s-\int_{0}^{t}(t-s) C(s) y d s\right) \leq \frac{t^{2}}{2} M q\left(A x_{n}-y\right) .
$$

And since $\lim _{n \longrightarrow \infty} A x_{n}=y, \frac{t^{2}}{2} M q\left(A x_{n}-y\right) \longrightarrow 0$, as $n \longrightarrow \infty$. 
Similarly, we obtain the result for $t \in \mathbf{R}^{-}$. Then,

$$
C(t) x-x=\int_{0}^{t}(t-s) C(s) y d s
$$

which implies $x \in D(A)$ and $A x=y$.

Hence, $A$ is closed.

\section{Strongly continuous cosine families in Quojection :}

Exapmle 1. we give an example of uniformly continuous cosine family on Fréchet space whose infinitesimal generator is not everywhere defined.

A matrix $\left(a_{n}(i)\right)_{i, n \in \mathbf{N}}$ of non-negative numbers is called a Köthe matrix if it satisfies the following conditions :

1. $\forall i \in \mathbf{N}, \exists n \in \mathbf{N}$ such that $: a_{n}(i)>0$.

2. $a_{n}(i) \leq a_{n+1}(i), \forall i, n \in \mathbf{N}$.

Let $k=\left(a_{n}(i)\right)_{i, n \in \mathbf{N}}$ be a Köthe matrix satisfies :

$$
a_{n}(i) \geq 1 \quad \text { and } \quad \sum_{i \in \mathbf{N}} \frac{a_{n}(i)}{a_{n+1}(i)}<\infty, \forall n \in \mathbf{N} .
$$

We consider the space

$$
\lambda_{1}(K)=\left\{x=\left(x_{i}\right)_{i \in \mathbf{N}} \in \mathbf{C}^{\mathbf{N}}: p_{n}(x)=\sum_{i \in \mathbf{N}} a_{n}(i)\left|x_{i}\right|<\infty, \forall n \in \mathbf{N}\right\} .
$$

$\lambda_{1}(K)$ equipped with the family of semi-norms $\left\{p_{n}\right\}_{n=1}^{\infty}$ is a nuclear Fréchet space [7].

Let $\left(\mu_{i}\right)_{i \in \mathbf{N}}$ be a sequence of real numbers such that each $\mu_{i}>0$ and $\lim _{i \longrightarrow \infty} \mu_{i}=\infty$.

For each $t \in \mathbf{R}$, define a linear operator $C(t)$ on $\lambda_{1}(K)$ by :

$$
C(t) x=\left(\operatorname{Cos}\left(\sqrt{\mu_{i}} t\right) x_{i}\right)_{i=1}^{\infty} \quad, \quad x \in \lambda_{1}(K) .
$$

Let $x \in \lambda_{1}(K)$, and $\varepsilon>0$; for given $n \in \mathbf{N}, \exists i_{0} \in \mathbf{N}$ such that :

$$
\sum_{i>i_{0}} a_{n}(i)\left|x_{i}\right|<\frac{\varepsilon}{4}
$$


On the other hand, $\exists \eta>0$ such that $\forall h \in \mathbf{R}^{*}$, with $|h|<\eta$, we have :

$$
\sum_{i \leq i_{0}}\left|\cos \left(\sqrt{\mu_{i}}(t+h)\right)-\cos \left(\sqrt{\mu_{i}} t\right)\right| a_{n}(i)\left|x_{i}\right|<\frac{\varepsilon}{2} .
$$

Then, for all $h \in \mathbf{R}^{*}$, with $|h|<\eta$ we have :

$$
\begin{aligned}
p_{n}(C(t+h) x-C(t) x) & =\sum_{i \in \mathbf{N}}\left|\cos \left(\sqrt{\mu_{i}}(t+h)\right)-\cos \left(\sqrt{\mu_{i}} t\right)\right| a_{n}(i)\left|x_{i}\right| \\
& \leq \frac{\varepsilon}{2}+2 \sum_{i>i_{0}} a_{n}(i)\left|x_{i}\right| \leq \frac{\varepsilon}{2}+2 \frac{\varepsilon}{4}=\varepsilon
\end{aligned}
$$

Then $\{C(t)\}_{t \in \mathbf{R}}$ is a strongly continuous cosine family on $\lambda_{1}(K)$, and hence, it is also uniformly continuous as $\lambda_{1}(K)$ is Montel, since it is nuclear [8].

Let $A$ the infinitesimal generator of the family $\{C(t)\}_{t \in \mathbf{R}}$, then we have:

$A x=\left(-\mu_{i} x_{i}\right)_{i=1}^{\infty}$, and $D(A)=\left\{x \in \lambda_{1}(K)\right.$; tel que $\left.\left(-\mu_{i} x_{i}\right)_{i=1}^{\infty} \in \lambda_{1}(K)\right\}$.

Indeed, let $x \in D(A)$, i-e $\lim _{t \rightarrow 0} \frac{2}{t^{2}}(C(t) x-x)$ exist on $X$. Thus $\exists y \in \lambda_{1}(K)$ such that for all $n \in \mathbf{N}$ we have: $\lim _{t \rightarrow 0} \sum_{i=0}^{\infty} a_{n}(i) \mid\left(\frac{2}{t^{2}}\left(\operatorname{Cos}\left(\sqrt{\mu_{i}} t\right) x_{i}-x_{i}\right)-\right.$ $\left.y_{i}\right) \mid=0$.

Hence $y_{i}=\lim _{t \longrightarrow 0}\left(\frac{2}{t^{2}}\left(\operatorname{Cos}\left(\sqrt{\mu_{i}} t\right) x_{i}-x_{i}\right)=-\mu_{i} x_{i}, \forall i \in \mathbf{N}\right.$.

Therefore,

$D(A) \subset\left\{\left(x_{n}\right)_{n \in \mathbf{N}} \in \lambda_{1}(K) ;\left(-\mu_{i} x_{i}\right)_{i \in \mathbf{N}} \in \lambda_{1}(K)\right\}$, and $A\left(x_{i}\right)_{i \in \mathbf{N}}=\left(-\mu_{i} x_{i}\right)_{i \in \mathbf{N}}$.

Conversely, let $x \in \lambda_{1}(K)$ such that $\left(-\mu_{i} x_{i}\right)_{i \in \mathbf{N}} \in \lambda_{1}(K)$, let $n \in \mathbf{N}$ then for all $t \in \mathbf{R}^{*}$ we have :

$$
p_{n}\left(\frac{2}{t^{2}}(C(t) x-x)+\left(\mu_{i} x_{i}\right)_{i \in \mathbf{N}}\right)=\sum_{i=0}^{\infty} a_{n}(i)\left|\frac{2}{\mu_{i} t^{2}}\left(\cos \left(\sqrt{\mu_{i}} t\right)-1\right)+1\right|\left|\mu_{i} x_{i}\right| .
$$

Put $g(t)=\frac{2(\cos (t)-1)}{t^{2}}+1, t \in \mathbf{R}$. The function $g$ is even, then it is enough to study $g$ on $\mathbf{R}^{+}$. Since $1-\frac{t^{2}}{2} \leq \cos (t) \leq 1$ for all $t \in \mathbf{R}^{+}$, which means $0 \leq g(t) \leq 1 \forall t \in \mathbf{R}$.

Hence :

$$
p_{n}\left(\frac{2}{t^{2}}(C(t) x-x)+\left(\mu_{i} x_{i}\right)_{i \in \mathbf{N}}\right) \leq \sum_{i=0}^{\infty} a_{n}(i)\left|\mu_{i} x_{i}\right|<\infty .
$$


Therefore,

$$
\begin{gathered}
\lim _{t \longrightarrow 0} p_{n}\left(\frac{2}{t^{2}}(C(t) x-x)+\left(\mu_{i} x_{i}\right)_{i \in \mathbf{N}}\right) \\
=\sum_{i=0}^{\infty} \lim _{t \longrightarrow 0}\left|\frac{2}{\mu_{i} t^{2}}\left(\cos \left(\sqrt{\mu_{i}} t\right)-1\right)+1\right| a_{n}(i)\left|\mu_{i} x_{i}\right|=0 .
\end{gathered}
$$

Then

$$
\begin{gathered}
\left\{\left(x_{n}\right)_{n \in \mathbf{N}} \in \lambda_{1}(K) ;\left(-\mu_{i} x_{i}\right)_{i \in \mathbf{N}} \in \lambda_{1}(K)\right\} \subset D(A), \text { and } \\
A\left(x_{i}\right)_{i \in \mathbf{N}}=\left(-\mu_{i} x_{i}\right)_{i \in \mathbf{N}} .
\end{gathered}
$$

Finally, for each $n \in \mathbf{N}$ we put $\mu_{i}=\sum_{n=1}^{i} a_{n}(i), \forall i \in \mathbf{N}$.

we have $\mu_{i} \longrightarrow \infty$ and the sequence $\frac{1}{\mu}=\left(\frac{1}{\mu_{i}}\right)_{i \in \mathbf{N}} \in \lambda_{1}(K)$, because for all $m \in \mathbf{N}$,

$$
\begin{aligned}
p_{m}\left(\frac{1}{\mu}\right)=\sum_{i \in \mathbf{N}} a_{m}(i) \frac{1}{\mu_{i}} & =\sum_{i=1}^{m} a_{m}(i) \frac{1}{\mu_{i}}+\sum_{i=m+1}^{\infty} \frac{a_{m}(i)}{\sum_{n=1}^{i} a_{n}(i)} \\
& \leq \sum_{i=1}^{m} a_{m}(i) \frac{1}{\mu_{i}}+\sum_{i=m+1}^{\infty} \frac{a_{m}(i)}{a_{m+1}(i)}<\infty .
\end{aligned}
$$

but, $\left(-\mu_{i} \cdot \frac{1}{\mu_{i}}\right)_{i \in \mathbf{N}}=(-1)_{i \in \mathbf{N}} \notin \lambda_{1}(K)$, then $\frac{1}{\mu} \notin D(A)$ and $D(A) \neq \lambda_{1}(K)$.

Proposition 5. Suppose that $X$ is a Fréchet space which contains a complemented copy of some nuclear Köthe sequence space $\lambda_{1}(K)$. Then there exists an equicontinuous, uniformly continuous cosine family in $X$ whose infinitesimal generator is not everywhere defined.

Proof. Let $P: X \longrightarrow X$ be any projection satisfying $\operatorname{Im}(P)=\lambda_{1}(K)$, and define $\operatorname{Ker}(P)=Y$. According to Example 1., for each $t \in \mathbf{R}$, define a linear operator $C_{1}(t)$ on $\lambda_{1}(K)$ by :

$$
C_{1}(t) x=\left(\operatorname{Cos}\left(\sqrt{\mu_{i}} t\right) x_{i}\right)_{i=1}^{\infty} \quad, \quad x \in \lambda_{1}(K) .
$$

The family $\left\{C_{1}(t)\right\}_{t \in \mathbf{R}}$ is an equicontinuous, uniformly continuous cosine family in $\lambda_{1}(K)$.

Let $A \in \mathcal{L}(Y)$, such that $\left\{A^{n}\right\}_{n=1}^{\infty}$ is equicontinuous in $\mathcal{L}(Y)$. i.e. $\forall p \in$ $\Gamma_{X}, \exists q \in \Gamma_{X}, \exists M \geq 0$, such that :

$$
p\left(A^{n} x\right) \leq M q(x), \quad \forall n \in \mathbf{N}, \quad \forall x \in X .
$$


for each $t \in \mathbf{R}$, define a linear operator $C_{2}(t)$ on $Y$ by :

$$
C_{2}(t) x=\frac{e^{-t} e^{t A}+e^{t} e^{-t A}}{2}=\frac{e^{-t}}{2} \sum_{n=0}^{\infty} \frac{t^{n}}{n !} A^{n}+\frac{e^{t}}{2} \sum_{n=0}^{\infty} \frac{(-t)^{n}}{n !} A^{n} \quad, \quad x \in Y .
$$

According to $(*)$, the family $\left\{C_{2}(t)\right\}_{t \in \mathbf{R}}$ is an equicontinuous, uniformly continuous cosine family in $Y$.

Then, the family $\{C(t)\}_{t \in \mathbf{R}}$ of continuous linear operators in $X$ defined via:

$$
C(t) x=C_{1}(t) P x+C_{2}(t)(I-P) x, \quad t \in \mathbf{R}, \quad x \in X .
$$

is an equicontinuous, uniformly continuous cosine family in $\mathrm{X}$ whose infinitesimal generator is not everywhere defined.

Definition 3. A Fréchet space $X$ is a quojection if it is the projective limit of a projective system of Banach spaces $\left(\left(X_{n},\|\cdot\|_{n}\right)_{n=1}^{\infty},\left(\Pi_{n}^{m}\right)_{n \leq m}\right)$, with $\Pi_{n}^{m}: X_{m} \longrightarrow X_{n}$ is surjective, $\forall m \geq n$.(i.e $\left.X=\operatorname{Proj}_{n}\left(X_{n}, \Pi_{n}^{m}\right)\right)$.

Theorem 1. Let $X$ be a quojection.

The infinitesimal generator of every uniformly continuous cosine family is continuous, (i.e. $A \in \mathcal{L}(X)$ ). Moreover, we have :

$$
C(t) x=\sum_{k=1}^{\infty} \frac{t^{2 k}}{(2 k) !} A^{k} x, \quad \forall x \in X .
$$

Proof. Since $X$ is a quojection, then it is the projective limit of projective system $\left(\left(X_{n},\|\cdot\|_{n}\right)_{n=1}^{\infty},\left(\Pi_{n}^{m}\right)_{n \leq m}\right)$ of Banach spaces, with $\Pi_{n}^{m}: X_{m} \longrightarrow$ $X_{n}$ is surjective, $\forall n \leq m \in \mathbf{N}$, and we have $\Pi_{m}: X \longrightarrow X_{m}$ is sujective, $\forall m \in \mathbf{N}$.

$X$ is tonnelé, according to proposition 3. the family $\{C(t)\}_{t \in \mathbf{R}}$ is locally equicontinuous.

i.e. : for all $t_{0}>0$ fix, $\forall n \in \mathbf{N}, \exists m \in \mathbf{N}, m \geq n, \exists M>0$ such that :

$$
\left\|\Pi_{n}(C(t) x)\right\|_{n} \leq M\left\|\Pi_{m}(x)\right\|_{m}, \quad \forall t \in\left[-t_{0}, t_{0}\right], \quad \forall x \in X .
$$

On the other hand, for all $0<|t| \leq t_{0}$ we define the operator :

$$
\varphi_{t}(y)=\frac{2}{t^{2}} \int_{0}^{t}(t-s) C(s) y d s, \quad y \in X
$$


According to Corollary 1. we have $\varphi_{t}(y) \in D(A), \forall y \in X$, and $A \varphi_{t}(y)=\frac{2}{t^{2}}(C(t) y-y)$.

Since the family $\{C(t)\}_{t \in \mathbf{R}}$ is uniformly continuous, According to $\mathbf{R e}-$ mark 2. we have $: \varphi_{t} \longrightarrow I$, as $t \longrightarrow 0$, uniformly on bounded subsets of $X$.

For all $t \in\left[-t_{0}, t_{0}\right]$, we define the operator $\tilde{\varphi}_{t}$ on $X_{m}$ by :

$\tilde{\varphi}_{t}: \quad X_{m} \longrightarrow X_{n} \quad(m \geq n)$

$$
\Pi_{m}(x) \longrightarrow \Pi_{n}\left(\varphi_{t}(x)\right) \text {. }
$$

$\tilde{\varphi}_{t}$ is continuous, Indeed, let $x \in X$ we have :

$$
\left\|\tilde{\varphi}_{t}\left(\Pi_{m}(x)\right)\right\|_{n}=\left\|\Pi_{n}\left(\varphi_{t} x\right)\right\|_{n}=\left\|\frac{2}{t^{2}} \int_{0}^{t}(t-s) \Pi_{n}(C(s) x) d s\right\|_{n} .
$$

We obtain

$$
\left\|\tilde{\varphi}_{t}\left(\Pi_{m}(x)\right)\right\|_{n} \leq M\left\|\Pi_{m}(x)\right\|_{n}, \quad \forall t \in\left[-t_{0}, t_{0}\right] .
$$

Since $X$ is a quojection, $\exists B \in B(X)$ such that $B_{m} \subseteq \Pi_{m}(B)$, with $B_{m}$ is the unit ball of $X_{m}$ [3].

Thus, we have :

$$
\begin{aligned}
\sup _{z \in B_{m}}\left\|\tilde{\varphi}_{t}(z)-\Pi_{n}^{m}(z)\right\|_{n} & \leq \sup _{z \in \Pi_{m}(B)}\left\|\tilde{\varphi}_{t}(z)-\Pi_{n}^{m}(z)\right\|_{n} \\
& \leq \sup _{y \in B}\left\|\tilde{\varphi}_{t}\left(\Pi_{m}(y)\right)-\Pi_{n}^{m}\left(\Pi_{m}(y)\right)\right\|_{n} \\
& \leq \sup _{y \in B}\left\|\Pi_{n}\left(\varphi_{t}(y)-y\right)\right\|_{n}
\end{aligned}
$$

Since $\varphi_{t} \longrightarrow I$, as $t \longrightarrow 0$, uniformly on bounded subsets of $X, \tilde{\varphi}_{t}$ is uniformly converges on $B_{m}$ to $\Pi_{n}^{m}$.

Since the set of surjective operators is open in $\mathcal{L}\left(X_{m}, X_{n}\right)[9], \exists t_{1} \in \mathbf{R}^{*}$ such that $\tilde{\varphi_{t_{1}}}$ is surjective (for small $\left|t_{1}\right|$ ).

Let $n, m \in \mathbf{N}$, with $m \geq n$, such that (3.1) is verified, and we take $m_{0} \geq m$ again with (3.1) satisfies. 
Let $x \in X$, then $\exists y \in X$ such that $\Pi_{m}(x)=\tilde{\varphi_{t_{1}}}\left(\Pi_{m_{0}}(y)\right)=\Pi_{m}\left(\varphi_{t_{1}}(y)\right)$.

Which implies, $\Pi_{n}(C(h) x)=\Pi_{n}\left(C(h) \varphi_{t_{1}}(y)\right), h \in\left[-t_{0}, t_{0}\right]$. In particular, for $h=0$ we have $\Pi_{n}(x)=\Pi_{n}\left(\varphi_{t_{1}}(y)\right)$.

Thus, for all $0<|h| \leq t_{0}$ we have :

$$
\Pi_{n}\left(\frac{2}{h^{2}}(C(h) x-x)\right)=\Pi_{n}\left(\frac{2}{h^{2}}\left(C(h) \varphi_{t_{1}}(y)-\varphi_{t_{1}}(y)\right)\right) .
$$

Since,

$$
\left.A\left(\varphi_{t}(y)\right)=\frac{2}{t^{2}}(C(t) y-y)\right), \quad \forall y \in X
$$

Then, as $h \longrightarrow 0$ we obtain :

$$
\Pi_{n}(A x)=\Pi_{n}\left(\frac{2}{t_{1}^{2}}\left(C\left(t_{1}\right) y-y\right)\right) .
$$

Since $n$ is arbitrary, $A$ is defined for every $x \in X$. Moreover, $A$ is closed because $\{C(t)\}_{t \in \mathbf{R}}$ is locally equicontinuous.

Hence, $A$ belongs to $\mathcal{L}(X)$.

For each $n \in \mathbf{N}^{*}$, we define the family $\left\{C_{n}(t)\right\}_{t \in \mathbf{R}}$, of operators, in $X_{n}$ by :

$$
C_{n}(t) \Pi_{n} x=\Pi_{n} C(t) x, \quad t \in \mathbf{R}, \quad x \in X .
$$

Each $\left\{C_{n}(t)\right\}_{t \in \mathbf{R}}, n \in \mathbf{N}$, form a strongly continuous cosine family in $X_{n}$. Actually, it is also uniformly continuous in $X_{n}$, indeed, let $B_{n}$ the unit ball of $X_{n}$, then $\exists B \in B(X)$ such that $B_{n} \subseteq \Pi_{n}(B)$, and we have :

$$
\sup _{x_{n} \in B_{n}}\left\|C_{n}(t) x_{n}-x_{n}\right\|_{n} \leq \sup _{\Pi_{n} x \in B_{n}}\left\|C_{n}(t) \Pi_{n} x-\Pi_{n} x\right\|_{n} \leq \sup _{x \in B}\left\|\Pi_{n}(C(t) x-x)\right\|_{n} .
$$

Since the family $\{C(t)\}_{t \in \mathbf{R}}$ is uniformly continuous in $X,\left\{C_{n}(t)\right\}_{t \in \mathbf{R}}$ is uniformly continuous in $X_{n}$. Hence $A_{n}$, the infinitesimal generator of $\left\{C_{n}(t)\right\}_{t \in \mathbf{R}}$, is a bounded operator in $X_{n}$, moreover, for each $n \in \mathbf{N}^{*}$, we have :

$$
A_{n} \Pi_{n} x=\Pi_{n} A x, \quad \forall x \in X .
$$

Since $X_{n}$ is a Banach space, the family $\left\{C_{n}(t)\right\}_{t \in \mathbf{R}}$ is written in the form :

$$
C_{n}(t) \Pi_{n} x=\sum_{k=1}^{\infty} \frac{t^{2 k}}{(2 k) !} A_{n}^{k} \Pi_{n} x, \quad \forall x \in X .
$$


Therefore,

$$
\Pi_{n} C(t) x=\Pi_{n} \sum_{k=1}^{\infty} \frac{t^{2 k}}{(2 k) !} A^{k} x, \quad \forall x \in X .
$$

Since $n$ is arbitrary, and $X=\operatorname{Proj}_{n}\left(X_{n}, \Pi_{n}^{m}\right)$,

$$
C(t) x=\sum_{k=1}^{\infty} \frac{t^{2 k}}{(2 k) !} A^{k} x, \quad \forall x \in X .
$$

Corollary 3. Let $X$ be a prequojection, then the infinitesimal generator of every uniformly continuous cosine family is continuous. (i.e. $A \in \mathcal{L}(X)$ ).

Proof. Since $X$ is prequojection, $X_{\beta}^{t t}$ is a quojection.

Let $\{C(t)\}_{t \in \mathbf{R}}$ be a uniformly continuous cosine family on $X$, then, according to Lemma 2.1. [1], the bi-dual operators $\left\{C(t)^{t t}\right\}_{t \in \mathbf{R}}$ form an uniformly continuous cosine family in $X_{\beta}^{t t}$.

Hence, According to Theorem 1, the infinitesimal generator of $\left\{C(t)^{t t}\right\}_{t \in \mathbf{R}}$, is belong to $\mathcal{L}\left(X_{\beta}^{t t}\right)$.

Actually, the infinitesimal generator of $\left\{C(t)^{t t}\right\}_{t \in \mathbf{R}}$, noted by $A^{t t}\left(D\left(A^{t t}\right)=\right.$ $\left.X_{\beta}^{t t}\right)$, is the bi-dual of infinitesimal generator $A$ of $\{C(t)\}_{t \in \mathbf{R}}$.

Since $A^{t t} / D(A)=A$, and $D(A)$ dense in $X$, it follows that $A$ is also everywhere defined, and $A \in \mathcal{L}(X)$.

\section{References}

[1] Albanese, A. A., Bonet, J. Ricker, W. J., Grothendieck spaces with the DunfordPettis property, Positivity, 14, pp. 145-164, (2010).

[2] Arendt, W., Batty, C. J. K., Hieber, M., Neubrander, F.: VectorValued Laplace Transforms and Cauchy Problems, Birkhauser, Basel, pp. 202-210, (2001) 
[3] Dierolf, S., Zarnadze, D. N., A note on strictly regular Fréchet spaces, Arch. Math., 42, pp. 549-556, (1984).

[4] Dieter L., Strongly continuous operator cosine functions, Functional analysis (Dubrovnik, 1981) Lecture Notes in Math., vol. 948, Springer, Berlin-New York, pp. 73-97 (1982)

[5] Fattorini, H. O.: Ordinary differential equations in linear topological spaces, II. J. Differential Equations 6, pp. 24-34, (1969)

[6] Jarchow, H., locally Convex Space, pp. 156, B. G. Teubner, stuttgart, (1981)

[7] Meise, R., Vogt, D, introduction to Function Analysis, pp.326-356, Clarendon press, Oxford, (1997)

[8] Schaefer, H. H, Topological Vector Spaces, pp. 101. Graduate textes in MAthematics, springer-verlag, Berlin Heidelberg New York, (1971)

[9] Serge L., Real and Functional Analysis, pp.396. third ed., Grad. Texts in Math., Vol. 142, Springer-Verlag, NewYork, (1993)

[10] Sova, M,. Cosine operator functions. Rozprawy Mat. 49, pp. 1-47, (1966).

\section{Rachid Ameziane Hassani}

Departement de Mathematiques, Faculté des Sciences Dhar-Mehraz, Université S. M. B. A.

B. P. 1769-Fes Atlas, Fes, Maroc

e-mail : rachid.amezianehassani@usmba.ac.ma

\section{Aziz Blali}

Ecole Normale Superieure, Departement de Mathematiques, Université S. M. B. A. B. P. 5206 Bensouda-Fes, Maroc

e-mail : aziz.blali@usmba.ac.ma 


\author{
Abdelkhalek El Amrani \\ Departement de Mathematiques, \\ Faculte des Sciences Dhar-Mehraz, \\ Universite S. M. B. A. \\ B. P. 1769-Fes Atlas, Fes, \\ Maroc \\ e-mail : abdelkhalek.elamrani@usmba.ac.ma \\ and
}

Khalil Moussaouja

Departement de Mathematiques,

Faculte des Sciences Dhar-Mehraz,

Universite S. M. B. A.

B. P. 1769-Fes Atlas, Fes,

Maroc

e-mail : khalil.moussaouja@usmba.ac.ma 